\title{
A Flying Spots Detection and Recovery Scheme Based on Hybrid-Waveform Recognition Method
}

\section{Qiyue Huang1, Changjin Hu², Zhenquan Sun², Xiaoning Kang1, Xuze Zhang1, Hao Wang1, Chong Zhao', Yali Ma ${ }^{3}$}

${ }^{1}$ Shaanxi Key Laboratory of Smart Grid (Xi'an Jiaotong University), Xi'an Jiaotong University, Xi'an, China

${ }^{2}$ Shaanxi Regional Electric Power Group CO., LTD, Xi'an, China

${ }^{3}$ Tianjin Power Transmission and Transformation Engineering Company, Tianjin, China

Email: 839539199@qq.com

How to cite this paper: Huang, Q.Y., Hu, C.J., Sun, Z.Q., Kang, X.N., Zhang, X.Z., Wang, H., Zhao, C. and Ma, Y.L. (2017) A Flying Spots Detection and Recovery Scheme Based on Hybrid-Waveform Recognition Method. Energy and Power Engineering, 9, 63-69.

https://doi.org/10.4236/epe.2017.94B008

Received: February 21, 2017

Accepted: March 30, 2017

Published: April 6, 2017

\begin{abstract}
Flying plots detection has been the focus of relay protection in power system for a long time. With the promotion of Smart substation in our country, the number of SV devices is greatly increased. Abnormal data (flying plot) caused by sampling device itself has brought tremendous pressure to the power system. The traditional flying plot detection algorithm has plenty of defects, such as low pertinence, low sensitivity and long sampling period. This paper proposes a new algorithm to identify flying plot by analyzing the wave form characteristics of sampling data. The traditional waveform recognition methods are combined in this algorithm. It has the concept of standard wave window and can distinguish flying plot in a short time. In addition, sine recovery algorithm is used to recover the flying plot. This paper uses PSCAD software to verify the validity of this algorithm. Simulation results show that the proposed method has high reliability.
\end{abstract}

\section{Keywords}

Hybrid-Waveform Recognition, Flying Plot, Abnormal Sampling, Sine Recovery, Standard Wave Window

\section{Introduction}

The detection of flying plot in power system is a key issue in the study of relay protection. In recent years, smart substations are promoted and new type protection devices based on smart grid greatly emerged. The number of SV equipments in access of protection devices greatly increased [1]. In addition to normal data 
noise, the flying plot in measurement data acquired by the information acquisition unit cannot be avoided, due to the objective reason. The flying spot data caused the great disturbance to the relay protection algorithm. It reduces the reliability and speed of traditional protection algorithm. It is the main reason of protective equipment malfunction. Therefore, it is very necessary to research the method of detecting fly spot [2].

This paper proposes a new flying plot detection and recovery scheme based on hybrid-waveform recognition method. This scheme classifies the flying pot. It detects and recovers flying plot by sine-recovery method and hybrid-waveform recognition method. Hybrid-waveform recognition algorithm combines the traditional integral and derivative waveform symmetry identification algorithm. It can compare and identify the shape of current by the concept of standard wave window. It can detect the flying plot data in a short time. This scheme increases the traditional protection methods' reliability and reduces the influence of flying plot and satisfies the requirements of protection devices.

\section{Hybrid-Waveform Recognition Method}

This paper proposes a hybrid-waveform recognition algorithm based on traditional methods. It combines the integral and derivative waveform symmetry identification algorithm. It identifies the differences between fault wave and normal wave with flying plot by standard wave window method. This method has both traditional methods' advantages and the characteristics of high reliability and speed [3].

\subsection{Integral Type Waveform Symmetry Method}

The fault current measured by protection devices is similar to the standard sine wave when the line in fault. While bad data has the characteristic of disorder, the current wave with flying plot is different from standard sine wave. So the type of current can be judged by comparing the waveform of current in the first and second half cycle. Integral waveform symmetry principle is showed as below:

As it can be seen in Figure 1. A sampling period $A B C$ is divided into two section, $A B$ and $B C$. Inversing the first half period $A B$ by time axis to get the curve $A^{\prime} B^{\prime}$. The curve $B^{\prime} C^{\prime}$ can be gotten by translating $A^{\prime} B^{\prime}$ half cycle. Then we get the quadrilateral curve $B C C^{\prime} B^{\prime}$. Calculating its area as $S$, rectangular $B C C^{\prime} B^{\prime}$ area as $S_{t}$. Curve $A B$ combines with the time axis in first half cycle's area as $S_{+}$. Curve $B C$ combines with the time axis in second half cycle's area is $S_{+}$. Defined waveform a symmetry factor as below:

$$
K_{\text {sym }}=\frac{\left|S-S_{t}\right|}{\max \left(S_{+}, S_{-}\right)}
$$

An ideal sine wave current is symmetry. So we can calculate that $K_{\text {sym }}=0$. While the value of normal current with flying plots is bigger than zero.

\subsection{Derivative Type Waveform Recognition Method}

Document [4] proposed a waveform symmetry algorithm which is based on 


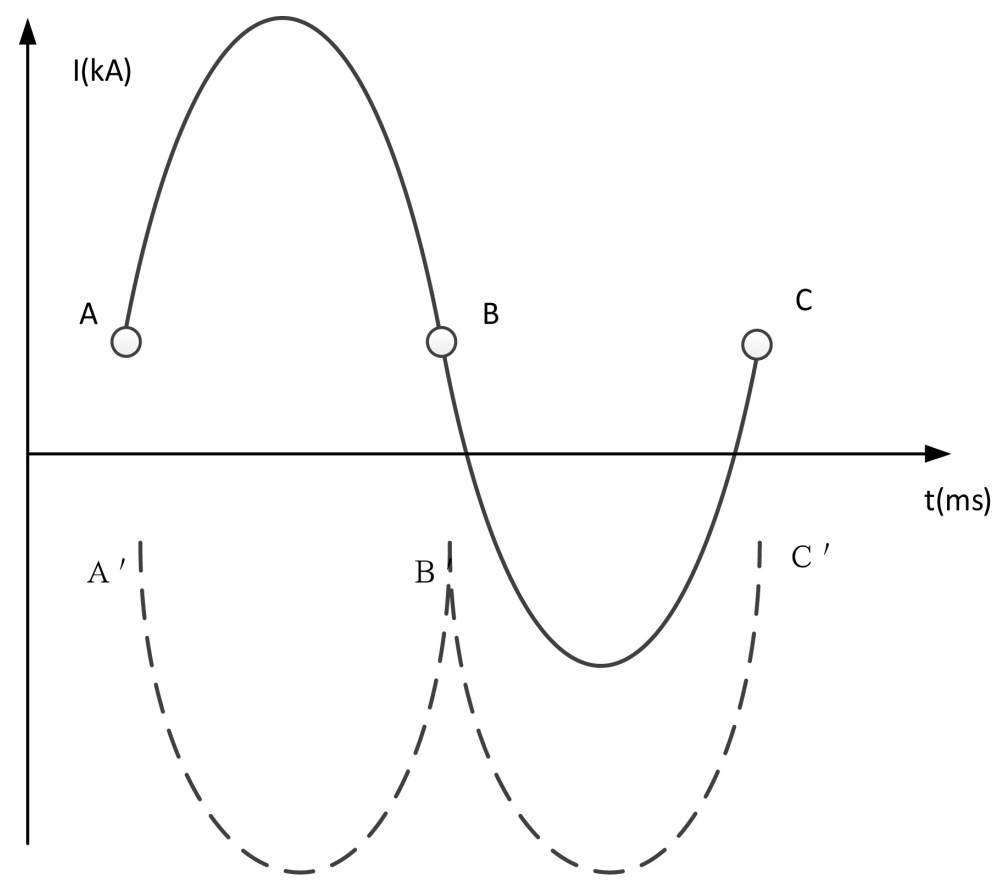

Figure 1. Figure of integeral waveform symmetry principle.

comparison of derivative of the current's half-wave symmetry. The basic idea of the algorithm is obtaining a differential current without DC component by derivate method. Then it compares the first-half and second-half waveform of derivative current to check if it is symmetry. The formula is showed as below:

$$
\frac{I_{i}+I_{i+180^{\circ}}}{I_{i}-I_{i+180^{\circ}}} \leq K_{\text {sym }}
$$

In this formula, $I_{i}$ is the value of a point in the first-half waveform. $I_{i+180}$ is the value of the corresponding point in the second-half waveform.

\subsection{Hybrid-Waveform Recognition Principle}

The waveform of fault current can be seen as standard sine wave, so its derivative waveform can also be seen as standard sine wave. The hybrid-waveform recognition algorithm derivates the measured current and units it. Then it compares the data with the standard sine wave. It can be determined that the current is fault current rather than normal current with flying plot when the shapes are similar. According to the integral waveform symmetry principle, giving the similarity coefficient of graphic as below:

$$
K_{\text {sym }}=\frac{\left|S_{1}-S_{2}\right|}{\max \left(S_{1}, S_{2}\right)}
$$

In this formula: $S_{1}$ is the integrated area of the derivative current in a certain time after normalized; $S_{2}$ is the integrated area of normalized sine wave in a certain time.

The integration time can be less than a period of time as long as they are equal. Therefore, this method has high precision and speed. 


\section{The Principle of Scheme}

This paper proposes a new scheme to detect and recover flying plot based on hybrid-waveform recognition method. The characteristic of the flying plot is easily seen from the shape of waveform. It is a sharp pulse or high frequency spot far away from the normal sampling value. The reason of the problems mainly is the environment of strong electromagnetic interference, lightning surge and collision problem of HASH algorithm.

Flying plot is a random and high value sampling point. It can be isolated point or continuous points without periodic feature. Flying plots can be divided into single flying plot and continuous flying plot. The scheme uses different methods to detect and restore flying plots in different type. The process of the scheme is showed as Figure 2.

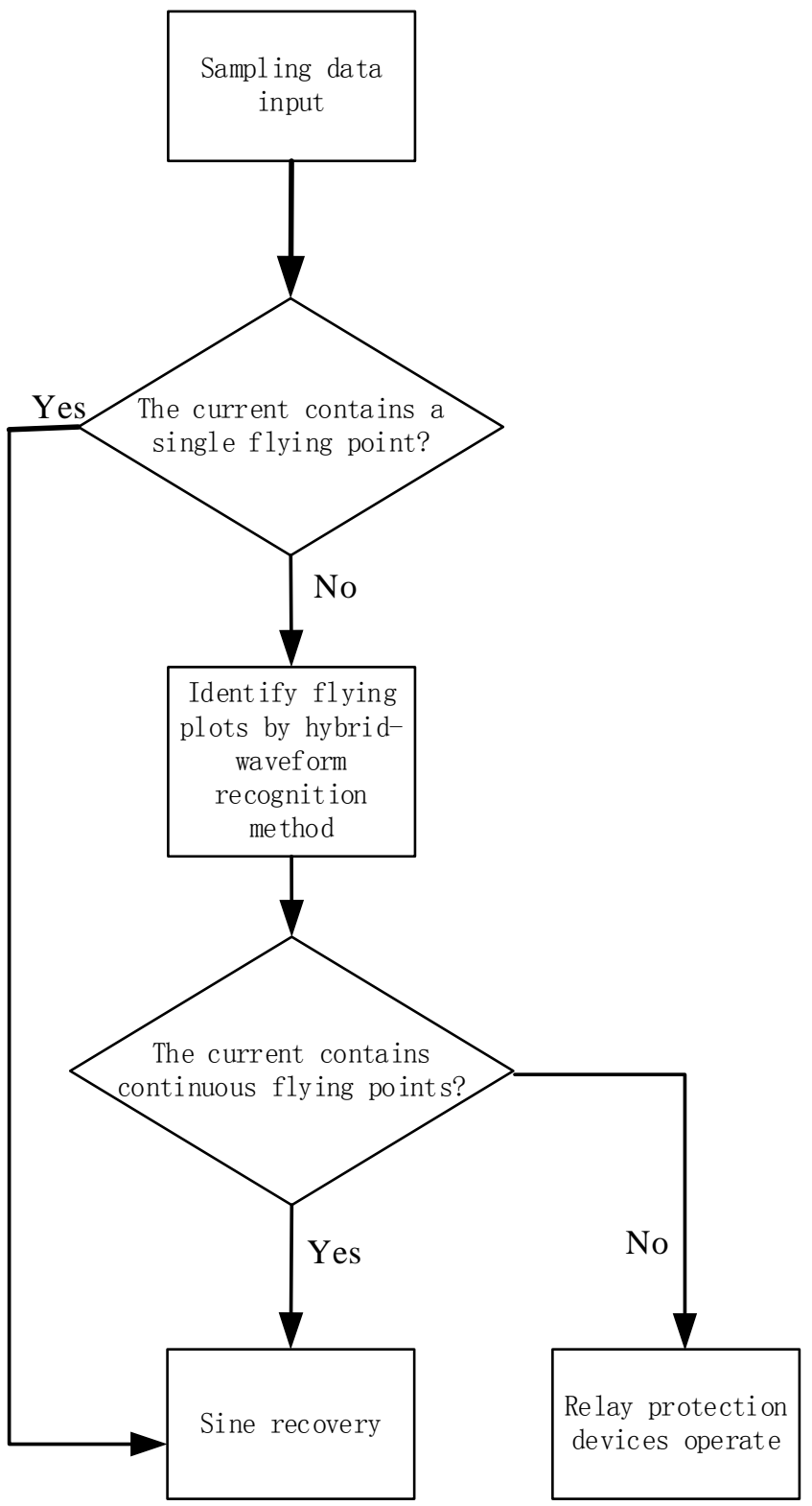

Figure 2. The flow diagram of the scheme. 


\subsection{The Detection of Single Flying Point}

When the measured current waveform has a single flying plot, the characteristic of flying plot is so obvious. The measured current cannot be fault current because the number of abnormal data is so less. Devices act normally. Therefore, the position of unknown data can be judged by the position of existed points. If the ideal position and actual position are the same, we can conclude that the data point is normal. If the results are opposite of what expected, the data point is a flying plot. This method can restore the flying plot by ideal position. It can reduce the influence of single flying point in relay protection.

\subsection{The Detection of Continuous Flying Points}

The single flying point detection method fails when measured current contains a series of flying points. It can't distinguish the fault current and normal current contains continuous flying points. The abnormal recovery of flying plots may cause the malfunction of protection device. It is necessary to distinguish the differences between fault current and normal current with flying plots. In the above, hybrid-waveform recognition method can be applied in fault current detection. The Equation (3) determines the shape of current in a short time to distinguish the type of current. Protection devices operate when the current is judged as fault current. It recovers measured current by sine recovery when the current is judged as normal current. This method can reduce the influence of multi-points in relay protection [5] [6].

\section{Results of Simulation}

In this paper, the proposed scheme is simulated and verified by PSCAD software. It constructs fault current and normal current with single a flying point and continuous flying points to test the correctness and accuracy of the proposed scheme. The results are showed as Figure 3-5.

It can be seen that the scheme can identify and recover the current when input current contains flying points. It doesn't recover the current when input current is fault current. The relay protection device can operate normally.

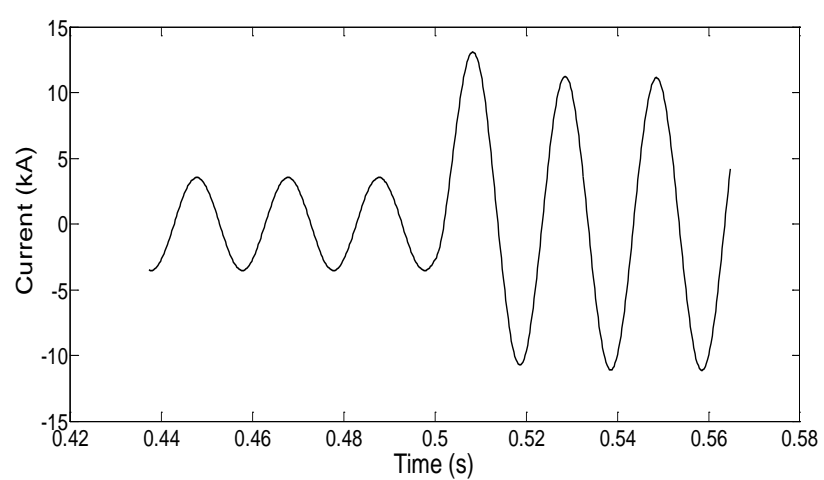

(a)

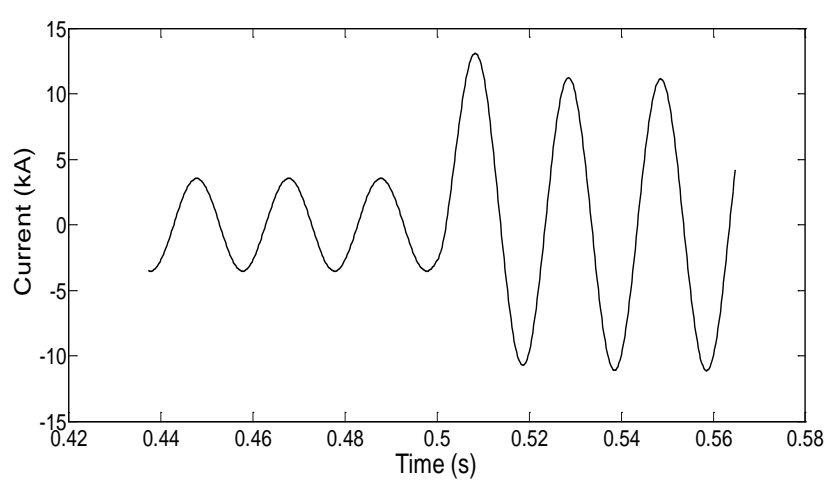

(b)

Figure 3. The results of fault current. (a) Measured current's wavform (b) Computed current's waveform. 


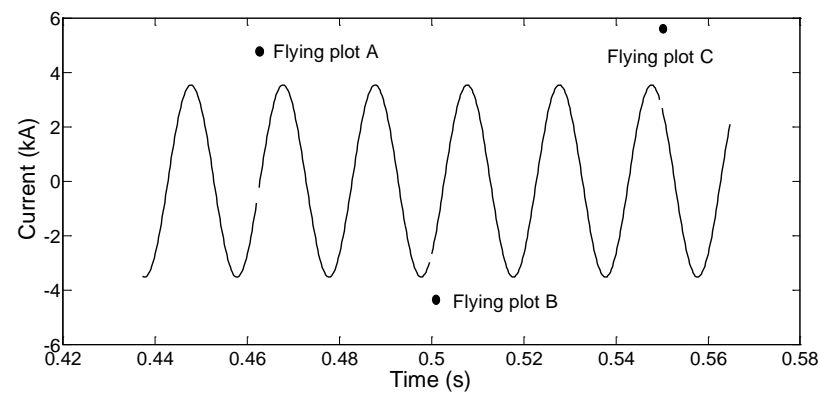

(a)

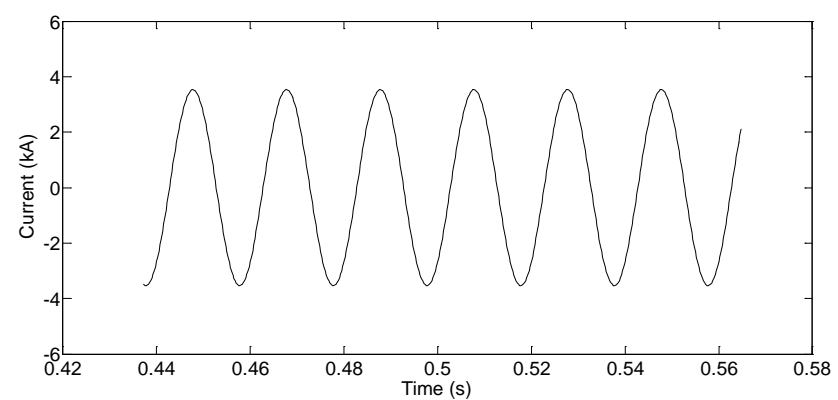

(b)

Figure 4. The results of current with single flying plot. (a) Measured current's waveform (b) Computed current's waveform.

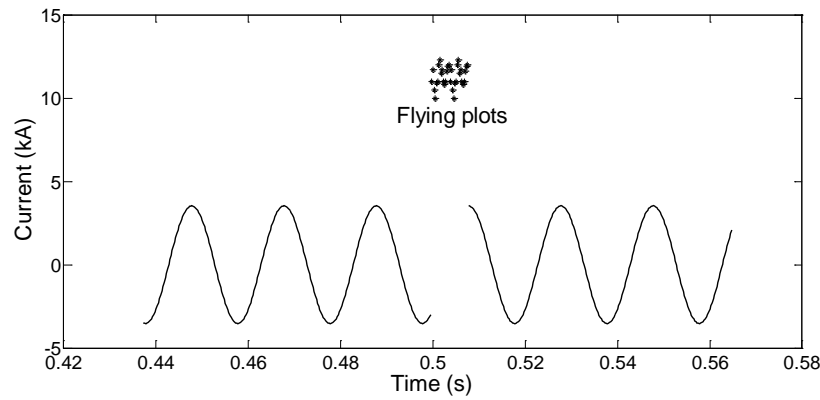

(a)

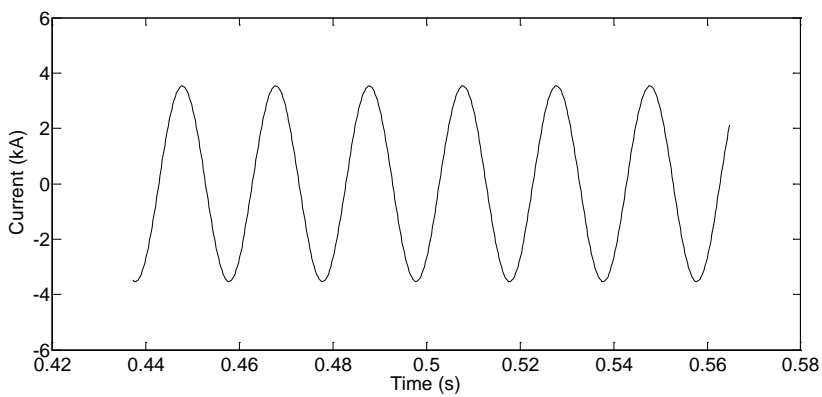

(b)

Figure 5. The results of current with continous flying plots. (a) Measured current's waveform (b) Computed current's waveform.

\section{Conclusion}

This paper describes the flying plot's classification and characteristic. It also introduces their bad effect on relay protection. Analyzing domestic and foreign well-known scholars' study on detection of flying plots, the existing methods have plenty of problems. These defects greatly affect the reliability and speed of relay protection in practical engineering. This paper presents a new scheme based on hybrid-waveform recognition algorithm to detect flying plots. The algorithm combines the traditional waveform recognition methods. It has high reliability and accuracy. This scheme can identify the shape of waveform by using different strategy to determine the type of current. It can identify flying plots and recover them. Relay protection devices can operate when input current is judged as fault current. The simulation of PSCAD software shows that this scheme has high reliability. With the promotion of substation in our country, the scheme has good application ability and research potential [7].

\section{References}

[1] Wu, W.J. and Zhang,J.W. (2015) A flying Spot Detection Algorithm For Continuous Sampling Based on Removable Discontinuous Points. Power system Protection and Control,4, 18-24.

[2] Wang, J.Y., Zhang, X.L. and Ai, S.Y. (2015) Research on Test Plan and Features of Abnormal Big Date in SV Sampling.Electric Power Survey and Design,5, 47-52.

[3] Zhao, L., Qian, Y.C. and Liu, H.J. (2010) Abnormal Data Resisting Method in Digital Substation. Automation of Electric Power System,34, 97-99. 
[4] Zhang, S. (2012) Transformer Magnetizing Inrush Current Identification Method Based on Energy Component. Power System Protection and Control, 17, 121-126.

[5] Zhang, Y.C. (2006) Bad data detection and Identification in Power System. Southwest Jiaotong University, Chengdu.

[6] Huang, Y.Q., Xiao, J. and Li, Y.F. (2006) A New Method To Detect And Identify Bad Data Based on Correlatively of Measured Data in Power System. Power System Technology, 30, 70-74.

[7] Yang, Z. (2011) Analysis and Solution of The Flying Plot in the Smart Substation.Xinjiang Electric Technology,1.

Submit or recommend next manuscript to SCIRP and we will provide best service for you:

Accepting pre-submission inquiries through Email, Facebook, LinkedIn, Twitter, etc. A wide selection of journals (inclusive of 9 subjects, more than 200 journals)

Providing 24-hour high-quality service

User-friendly online submission system

Fair and swift peer-review system

Efficient typesetting and proofreading procedure

Display of the result of downloads and visits, as well as the number of cited articles Maximum dissemination of your research work

Submit your manuscript at: http://papersubmission.scirp.org/

Or contact epe@scirp.org 